\section{Safety of an Accelerated Build-up Phase With Pollen Allergoids: A Retrospective Study}

Caruso $\mathrm{C}^{1}$, Borgonovo $\mathrm{L}^{2}$, Bramé $\mathrm{B}^{3}$, Cocconcelli $\mathrm{A}^{4}$, Colantuono $\mathrm{S}^{5}$, Piantanida $\mathrm{M}^{6}$, Scarpa $\mathrm{S}^{7}$, Zisa $\mathrm{G}^{8}$, Romano $\mathrm{A}^{1}$

${ }^{1}$ Allergy Unit - Fondazione Policlinico Gemelli -Presidio Columbus, Rome, Italy

${ }^{2}$ Allergy and Clinical Immunology Department, ASST

Fatebenefratelli Sacco, Milan, Italy

${ }^{3}$ Clinical Allergy and Immunology Unit, Legnano Hospital, Legnano, Italy

${ }^{4}$ Allergy Outpatient Clinic, AUSL Reggio Emilia, Reggio Emilia, Italy

${ }^{5}$ Clinical Medicine Department and Molecular Medicine Department, Sapienza University, Rome, Italy

${ }^{6}$ Clinical Allergy and Immunology Unit, Foundation IRCCS Ca' Granda, Ospedale Maggiore Policlinico, Milan, Italy

${ }^{7}$ Clinical Allergy and Immunology Unit, AUSL Parma, Parma, Italy

${ }^{8}$ Allergology and Immunology Unit, Novara Hospital, Novara, Italy

J Investig Allergol Clin Immunol 2018; Vol. 28(4): 283-284 doi: $10.18176 /$ jiaci.0269

Key words: Accelerated dose escalation. Allergoid. Safety. Subcutaneous immunotherapy. Tolerability.

Palabras clave: Incremento acelerado de dosis. Alergoide. Seguridad. Inmunoterapia Subcutánea. Tolerabilidad.

International guidelines suggest that the optimal treatment duration of allergen immunotherapy (AIT) is 3 years [1]. Like all long-term treatments, AIT is affected by poor adherence, which is mostly characterized by discontinuation of treatment before the end of the recommended period [2]. This problem affects both subcutaneous immunotherapy (SCIT) and sublingual immunotherapy (SLIT), even if some data suggest that adherence is better in patients receiving SCIT [3]. Other than clinical reasons, one of the most relevant factors for poor adherence to SCIT is the inconvenience related to the commute necessary to receive the allergy injections [4]; consequently, many attempts have been made to shorten treatment by reducing the number of injections [5].

The introduction of chemically modified allergens (high-dose hypoallergenic preparations, or allergoids) has made it possible to safely accelerate the build-up phase, and pollen allergoids have proved effective and safe with only 7 preseasonal injections [6]. Rush schedules with allergoids have also proven safe in previous studies [7,8], and a recent controlled study by Chaker et al [9] showed that an abbreviated build-up schedule with a 6-grass pollen allergoid extract (4 injections) was as safe and well-tolerated as the conventional 7-injection updosing schedule [9]. The aim of this retrospective study was to investigate the safety of the abbreviated build-up phase of pollen allergoids in real life.
We sent a questionnaire to allergy centers that adopted the shortened build-up schedule. The questionnaire included the following items: age, gender, symptoms, extract composition, maximum dose reached (yes/no), number of injections, local reactions $(>5 \mathrm{~cm},<10 \mathrm{~cm})$, large local reactions $(>10 \mathrm{~cm})$, and systemic reactions. The shortened regimen was administered with Allergovit (Allergopharma GmbH \& Co. KG), an AIT pollen extract that has been chemically modified with formaldehyde to produce an allergoid. Allergovit is specified in therapeutic units per $\mathrm{mL}(\mathrm{TU} / \mathrm{mL})$ and provided in 2 strengths (A, $1000 \mathrm{TU} / \mathrm{mL}$; and B, $10000 \mathrm{TU} / \mathrm{mL}$ ). The abbreviated updosing regimen consists of 4 injections, and the schedule is completed within 3 weeks only, while the conventional regimen comprises 7 injections. Patients received 2 weekly strength A injections and 2 strength B injections, with volumes of 0.2 and $0.6 \mathrm{~mL}$, respectively. In grass pollen Allergovit extracts, the maximum dose of $0.6 \mathrm{~mL}$ corresponds to a content of $25 \mu \mathrm{g}$ of grass group 5 allergens. The Figure shows a comparison of the classic and abbreviated build-up schedules.

We collected 156 cases of patients with seasonal allergic rhinoconjunctivitis who had been chosen for the short buildup; 85 of these patients also had asthma symptoms. Mean age was 36.0 years (range, 7-76), and there were 89 males and 67 females. The distribution by extract was as follows: grasses, 46; Parietaria, 36; ragweed, 26; birch, 13; mixed trees, 10; various mixtures, 25 .

All patients but one reached the maximum scheduled dose, and 138 patients $(88.5 \%)$ completed the treatment without reactions. A further 138 patients $(88.5 \%)$ also reached the maximum dose with the 4 scheduled injections. Eighteen patients required 1 extra injection, 9 for concomitant nontreatment-related events and 9 for adverse reactions.

Overall, 12 mild treatment-related local reactions $(>5 \mathrm{~cm}$, $<10 \mathrm{~cm}$ ) and 2 severe local reactions $(>10 \mathrm{~cm})$ were recorded ( $2.2 \%$ of the total 649 injections; $9.0 \%$ of patients). A severe local reaction was the reason why 1 patient did not reach the maximum dose. No systemic reactions were observed.

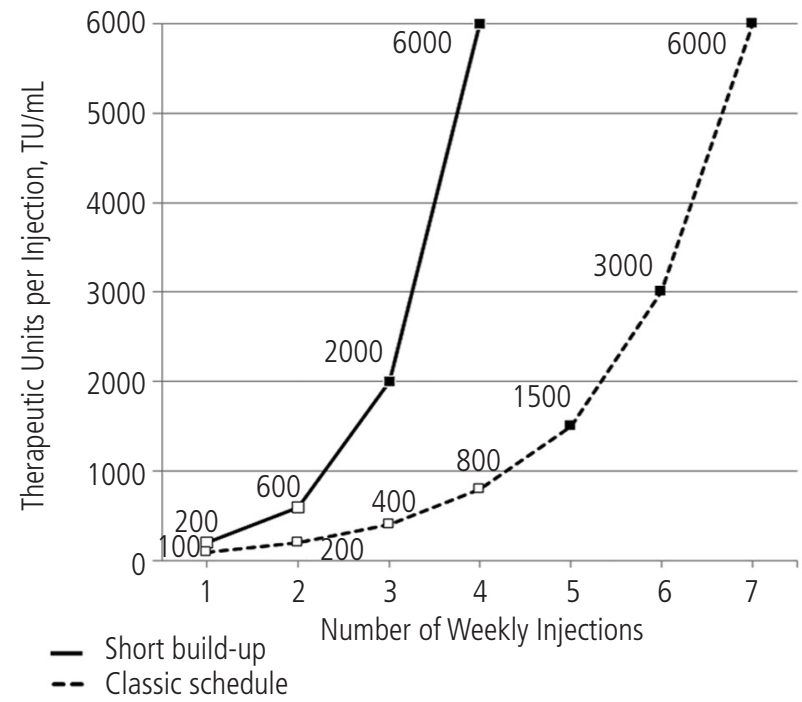

Figure. Comparison of the classic schedule and the short build-up. 
This study shows that the build-up phase of a high-dose hypoallergenic pollen allergoid can be safely shortened from the conventional 7 to only 4 injections, thus enabling the maintenance dose to be reached in only 3 weeks. This possibility has several advantages. Patients can benefit earlier from the treatment, since the clinical benefits of AIT appear very shortly after the maintenance dose is reached [5]. Shortening the escalation period can also address the problem of poor adherence, since adherence to SCIT is affected by logistic difficulties in attending the health care facility to receive the medication [4]. It is interesting to make a comparison of our findings with the results of the phase II randomized study by Chaker et al [9], who compared the safety and tolerability of the same short build-up schedule with the same 6-grass allergoid extract, which shared features with the classic 7-injection schedule [9]. While safety and tolerability were comparable between the 2 schedules, the authors observed local reactions $(>5 \mathrm{~cm})$ in $29.5 \%$ of patients in the abbreviated schedule and systemic reactions in $13.1 \%$ of the same group (WAO grades 1 and 2) [10]. Our results are considerably lower, although in the prospective phase, patients in the trial of Chaker et al remained under observation for 2 hours after the injection: in our retrospective, real-life survey, some late reactions may have been missed. In any case, the most relevant observation is that nearly all patients reached the maximum dose (155 out of 156), and a very high number of patients $(88.5 \%)$ completed the treatment with the 4 scheduled injections. Since these figures were registered in patient diaries, they are free from observation bias, thus proving the feasibility of the abbreviated schedule. Furthermore, Chaker et al only studied grass extract, while the safety of the short build-up in our survey was demonstrated with pollen extracts of various compositions (see above). In contrast with Chaker et al, who included only adult patients, our survey included 18 pediatric patients ( $<19$ years), of whom 12 were aged $<15$ years: no reactions were observed in this group. In any case, the number of reactions reported was too small to establish significant correlations (age, gender, extract composition, symptoms).

In conclusion, accelerating the build-up phase with hypoallergenic pollen allergoids is feasible and safe, reduces discomfort for patients, and increases adherence. Completing the treatment in only 3 weeks could attract more patients and make it possible to admit patients to AIT even close to the relevant pollen season. Such an approach will ultimately result in more patients enjoying the clinical benefits provided by AIT.

\section{Funding}

The authors declare that no funding was received for the present study.

\section{Conflicts of Interest}

The authors declare that they have no conflicts of interest.
2. Demoly P, Passalacqua G, Pfaar O, Sastre J, Wahn U. Patient engagement and patient support programs in allergy immunotherapy: a call to action for improving long-term adherence. Allergy Asthma Clin Immunol. 2016;12:34.

3. Egert-Schmidt AM, Kolbe JM, Mussler S, Thum-Oltmer S. Patients' compliance with different administration routes for allergen immunotherapy in Germany. Patient Prefer Adherence. 2014;8:1475-81.

4. Cox LS, Hankin C, Lockey R. Allergy immunotherapy adherence and delivery route: location does not matter. J Allergy Clin Immunol Pract. 2014;2(2):156-60.

5. Calabria CW. Accelerated immunotherapy schedules. Curr Allergy Asthma Rep. 2013;13(4):389-98.

6. Corrigan CJ, Kettner J, Doemer C, Cromwell O, Narkus A. Efficacy and safety of preseasonal-specific immunotherapy with an aluminium-adsorbed six-grass pollen allergoid. Allergy. 2005;60(6):801-7.

7. Pfaar $\mathrm{O}$, van Twuijver E, Hecker $H$, Boot JD, van Ree $R$, Klimek L. Accelerated up-dosing of subcutaneous immunotherapy with a registered allergoid grass pollen preparation. Int Arch Allergy Immunol. 2013;160(4):420-4.

8. Buczylko K, van der Werf JF, Boot D, van Ree R. Accelerated UpDosing of Subcutaneous Immunotherapy with a Registered Allergoid Birch Pollen Preparation. Int Arch Allergy Immunol. 2017;172(3):183-6.

9. Chaker AM, Al-Kadah B, Luther U, Neumann U, Wagenmann $M$. An accelerated dose escalation with a grass pollen allergoid is safe and well-tolerated: a randomized open label phase II trial. Clin Transl Allergy. 2015;6:4

10. Cox L, Larenas-Linnemann D, Lockey RF, Passalacqua G. Speaking the same language: The World Allergy Organization Subcutaneous Immunotherapy Systemic Reaction Grading System. J Allergy Clin Immunol. 2010;125(3):569-74, 574.e1574.e7.

Manuscript received February 8, 2018; accepted for publication April 20, 2018.

\author{
Cristiano Caruso
}

E-mail: Cristiano.caruso@policlinicogemelli.it

\section{References}

1. Jutel M, Agache I, Bonini S, Burks AW, Calderon M, Canonica $W$, et al. International consensus on allergy immunotherapy. J Allergy Clin Immunol. 2015;136(3):556-68. 